Supplement of

\title{
The novel HALO mini-DOAS instrument: inferring trace gas concentrations from airborne UV/visible limb spectroscopy under all skies using the scaling method
}

Tilman Hüneke et al.

Correspondence to: Tilman Hüneke (tilman.hueneke@iup.uni-heidelberg.de)

The copyright of individual parts of the supplement might differ from the CC BY 3.0 License. 


\section{Telescope pointing precision and accuracy}

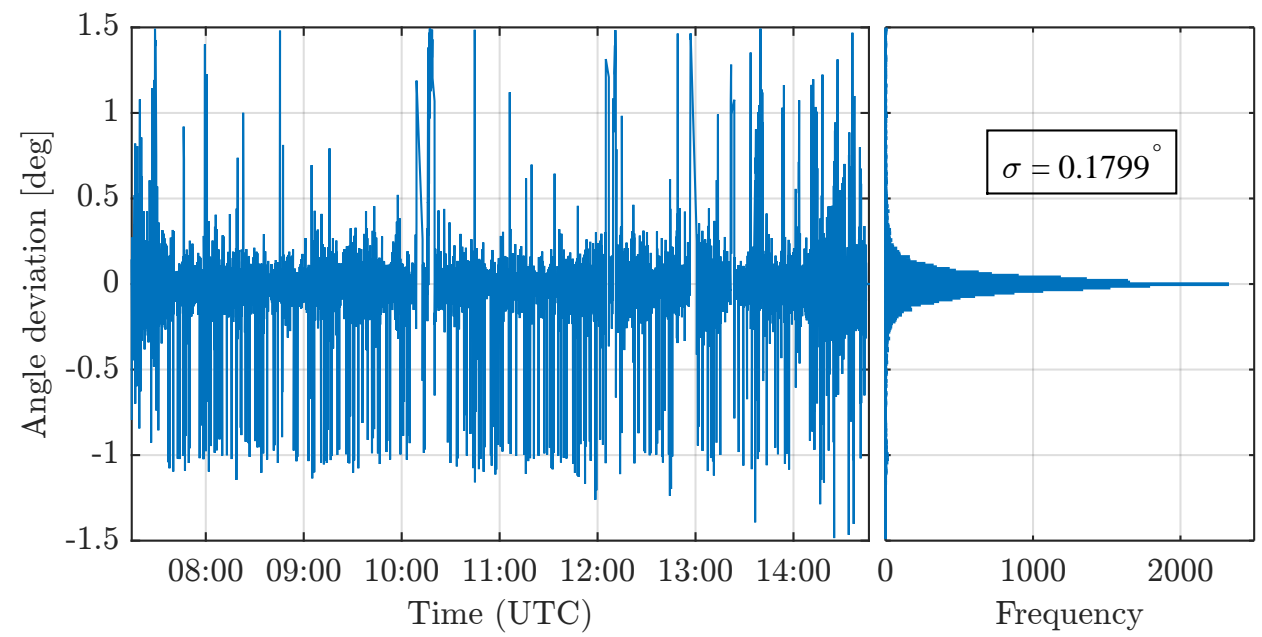

Figure 1: Deviation of the targeted elevation angle - taken from the aircraft's INS system - and the actual elevation angle for the HALO research flight of the TACTS mission on 30 August 2012. 

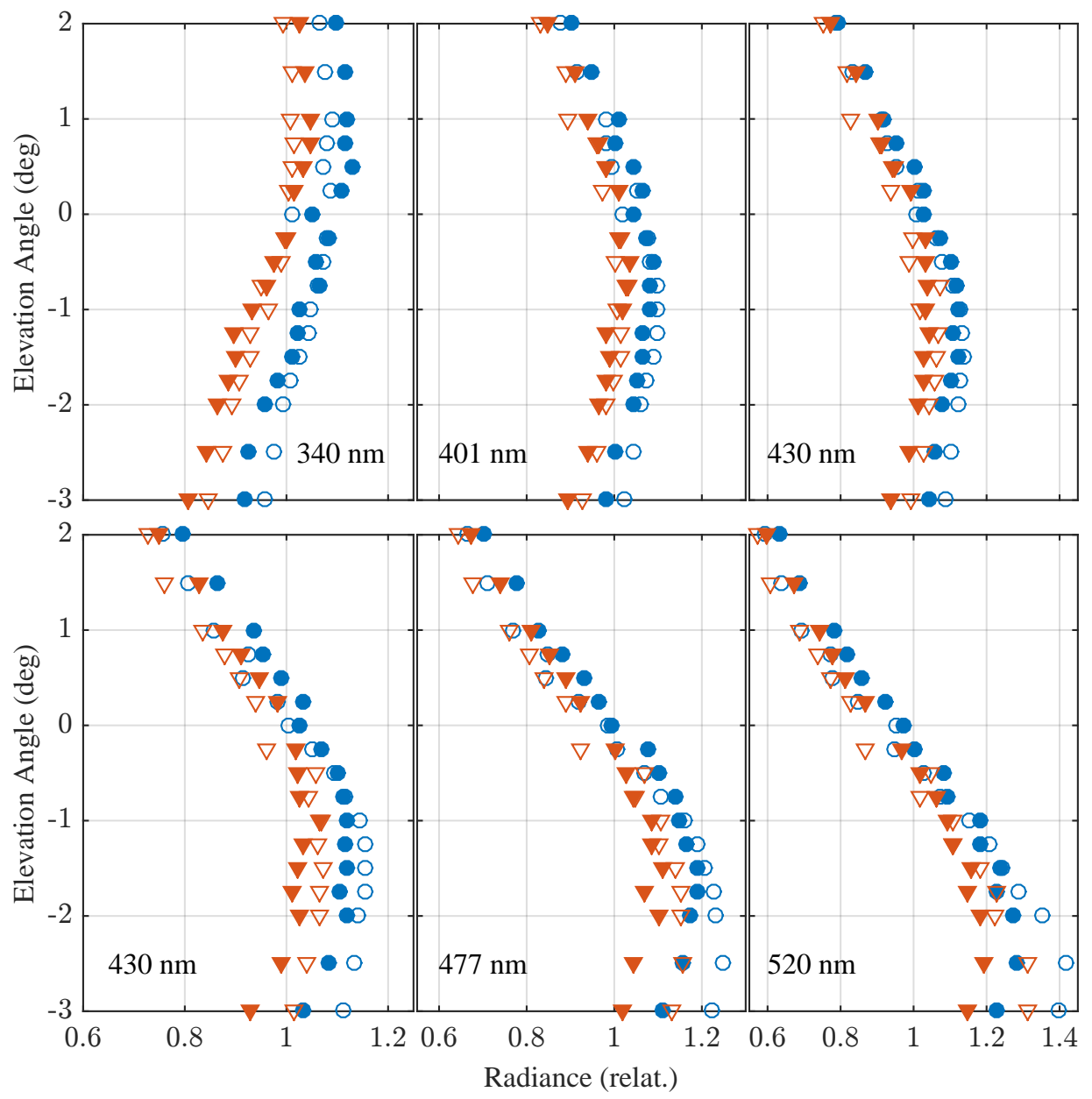

Figure 2: Comparison of modelled (filled symbols) and measured (unfilled symbols) relative radiances for a set of elevation angles between $+2^{\circ}$ and $-3^{\circ}$, recorded by the UV2 (upper panels) and VIS4 (lower panels) channels. Blue and red symbols indicate two subsequently recorded limb scans. Models and measurements are normalised to the mean of the respective dataset. The simulations employ the radiative transfer model (RTM) McArtim and climatological background aerosol profiles from satellite measurements. The measurements were carried out during the Polstracc science mission on 26 February 2016 at $13.7 \mathrm{~km}$ altitude, SZA $80^{\circ} \ldots 82^{\circ}$, SRAA $67^{\circ} \ldots 69^{\circ}$, and at $70^{\circ} \mathrm{N}, 47^{\circ} \mathrm{W}$. 


\section{$2 \quad \alpha_{R}$ random errors}

This section gives further details on the contributions to the random error $\Delta \alpha_{R}$, as described in section 3.7.1 of the paper.

\subsection{Uncertainty of $\alpha_{R}$ due to scattering by aerosols and clouds}

This section describes the RT model simulations to determine the uncertainty of $\alpha_{R}$ due to scattering by aerosols and clouds (section 3.7.1, $\Delta \alpha_{R}$, paragraph (a)).

For the cloud cover, the following is assumed (Figure 3): (a) A deck of marine strato-cumulus with a cloud base at $0.6 \mathrm{~km}$ and a cloud top at $1.2 \mathrm{~km}$, a scattering coefficient of $20 / \mathrm{km}$, an asymmetry factor (g) of $\mathrm{g}=0.85$, (b) $\mathrm{Cb}$ clouds with a cloud base at $0.6 \mathrm{~km}$ and a cloud top at $12.5 \mathrm{~km}$, a scattering coefficient of $40 / \mathrm{km}$ and a $\mathrm{g}=0.85$ (in the lower part) and $\mathrm{g}=0.7$ in upper part, and (c) cirrus clouds $(\mathrm{Ci})$ with a cloud base at $12 \mathrm{~km}$ and a cloud top at $12.5 \mathrm{~km}$, a scattering coefficient of $1 / \mathrm{km}$ and a $\mathrm{g}=0.7$. For all cloud types a single scattering albedo of $\omega_{0}=0.999$ is taken (for further details see Knecht (2015), section 4) and the clouds are assumed to be internally homogenous. This is justified to due the radiative smoothing within the cloud and our interest in modelling the disturbance of the radiative field and its effects on the ratio of the measured absorption for the scaling gas and target gas, in particular for the cloud free part of the atmosphere (Marshak and Davis, 2005). Further for the simulations the ground albedo of Pinker (1982) is taken and profiles of $\mathrm{O}_{4}$ and $\mathrm{CH}_{2} \mathrm{O}$ as shown in the insert of Figure 4 are assumed. Finally the clouds are arranged according to Figure 3. Then the positions and orientations of the limb telescope are randomly located in the surrogate cloud field.

For both gases the expected $\alpha$-factors are then simulated at $343 \mathrm{~nm}$ using the RT model McArtim (Deutschmann et al., 2011) for solar zenith angles of $14^{\circ}$ (given in blue in Figure 4) and $50^{\circ}$ (given in red in Figure 4). The frequency distributions show calculations based on the cloud scenario described above, and additionally the same calculations are carried out assuming clear skies (blue and red lines in Figure 4).

The calculations were performed in support for the interpretation of the measurement taken within the framework of the ACRIDICON campaign (Wendisch et al., 2016) which took place in the Amazon basin in fall 2014. There strong convection lead to cumulus and cumulus nimbus clouds which are largely varied in vertical and horizontal extent, providing an extreme test case to determine the sensitivity of the method as a function of cloud cover. Similar simulations were carried out for a different cloud scenario at $477 \mathrm{~nm}$, yielding similar results (see Knecht, 2015). 


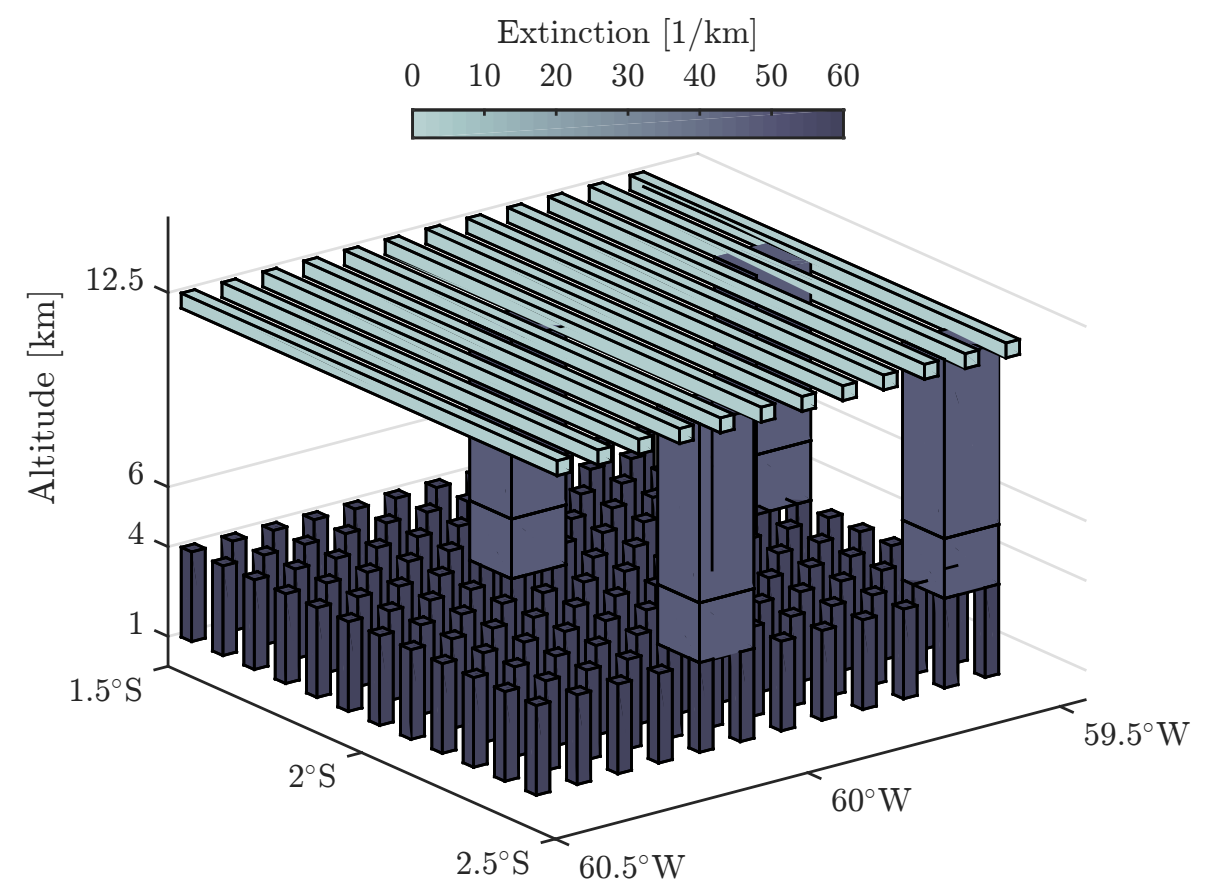

Figure 3: Surrogate cloud field assumed in the $\alpha$-ratio sensitivity study (for details see text) (Knecht, 2015). 


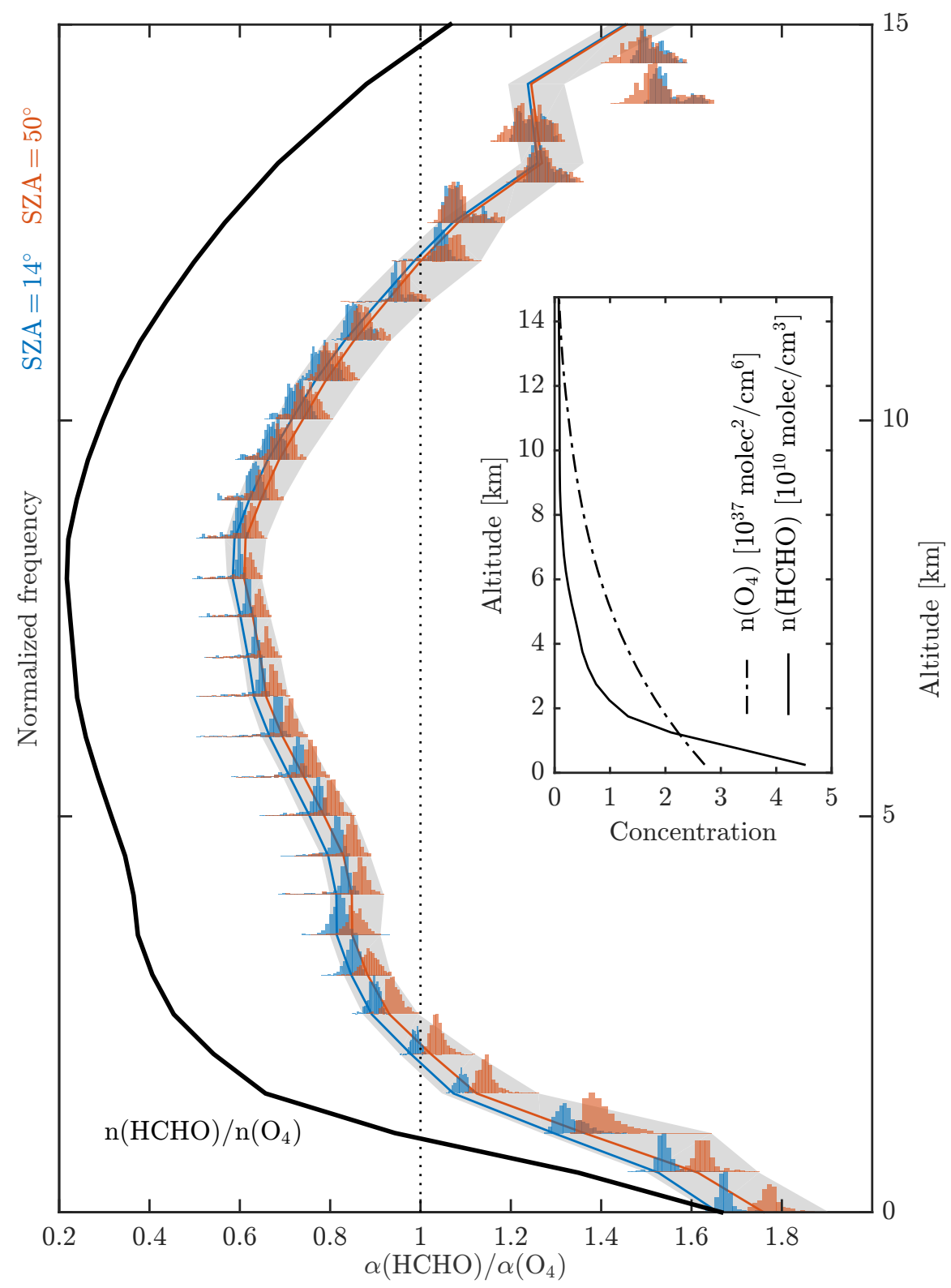

Figure 4: Simulated ratios of $\alpha_{\mathrm{HCHO}} / \alpha_{\mathrm{O}_{4}}$ factors for the assumed profiles of $\mathrm{HCHO}$ and $\mathrm{O}_{4}$ as shown in the insert. The concentration ratio $\mathrm{n}_{\mathrm{HCHO}} / \mathrm{n}_{\mathrm{O}_{4}}$ is shown as black profile. In the simulations the telescope is randomly placed and oriented in the azimuth direction but the elevation angle is kept constant at EA $=0^{\circ}$ to probe the atmosphere. Two sets of simulations are shown: A purely Rayleigh scattering atmosphere (blue/red lines and grey uncertainty range) and Rayleigh and Mie scattering in the surrogate cloud field shown in Figure 3 (blue/red frequency distributions of simulated Alpha factor ratios at the indicated altitude). Simulations are carried out for solar zenith angles of $15^{\circ}$ (blue) and $50^{\circ}$ (red). Adopted from (Knecht, 2015). 


\subsection{Small scale variability}

Figure 5 shows the comparison of CLaMS predictions and in situ measurements of $\mathrm{O}_{3}$, which indicates (a) a constant offset for parts of the data and (b) a scatter of in situ measurements around CLaMS predictions, which is interpreted as small scale variabilities which are not covered by the model. The in-situ measured $\mathrm{O}_{3}$ mixing ratios are smoothed from 100 seconds before start of each spectrum integration to 100 seconds after end of each spectrum integration in order to account for the horizontal sensitivity of each measurement due to multiple scattering.
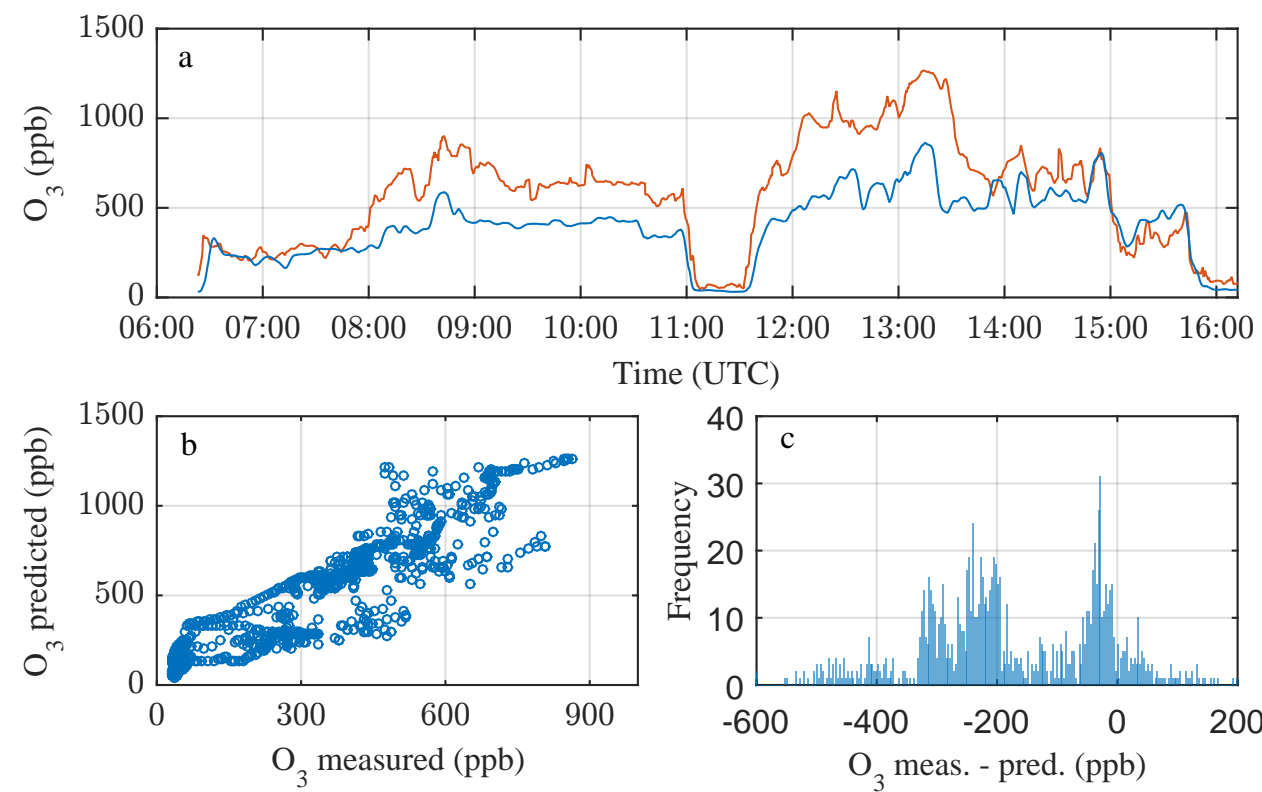

Figure 5: Comparison of in-situ measured (panel a, blue) and CLaMS predicted (panel a, red) $\mathrm{O}_{3}$ mixing ratio for the HALO flight from Cape Town to Antarctica on 13 September 2012. Panel b: Correlation of in-situ measured (Fairo) and CLaMS predicted $\mathrm{O}_{3}$ mixing ratios. Panel c: Frequency distribution of the difference in in-situ measured (Fairo) and CLaMS predicted $\mathrm{O}_{3}$. 


\subsection{Sum of random error contributions}

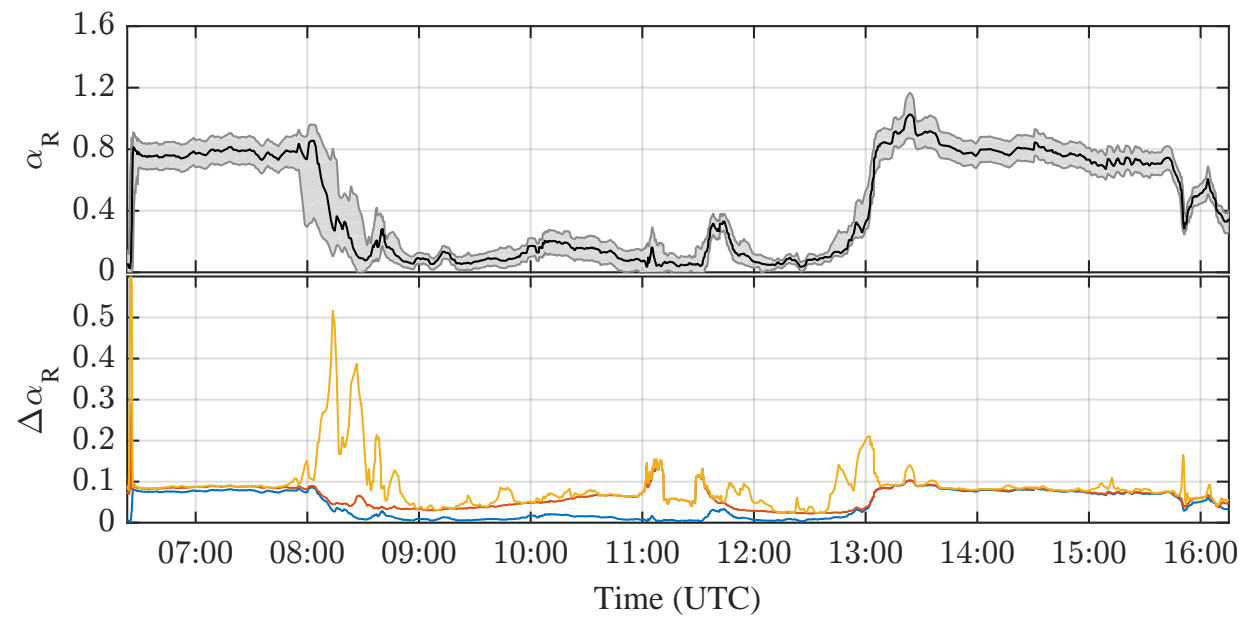

Figure 6: Summary of major random error contributions to $\Delta \alpha_{R}$ for the HALO flight on 13 September 2012. The $\alpha_{R}$ ratio (black line) and it's uncertainty $\Delta \alpha_{R}$ (grey shaded area) including all statistical components (top panel). Contribution of individual systematic errors to $\Delta \alpha_{R}$ subsequently added up (bottom panel): (a) The blue line show a 10\% uncertainty in the Mie extinction $\alpha_{R}$, (b) the red line shows the uncertainty due to small scale variability added to contribution (a) and the yellow line shows the vertical sampling uncertainty added to $(\mathrm{b})$. 


\section{$3 \quad \alpha_{R}$ systematic errors}

This section gives further details on the calculations to estimate potential systematic errors of the calculated $\alpha_{R}$ due to erronous predictions of the trace gas profile shapes by the employed CTMs, as described in section 3.7.2 of the paper. As mentioned there, the trace gas curtains are altered in such a way that (a) the concentration of $\mathrm{O}_{3}$ at flight altitude changed to match the in situ measured concentration, and (b) an altitude offset is calculated by comparing in situ measured and predicted $\mathrm{N}_{2} \mathrm{O}$, and the curtains of $\mathrm{O}_{3}$ and $\mathrm{NO}_{2}$ are both shifted accordingly.

Figure 7 shows how $\alpha_{\mathrm{R}}$ and the inferred $\left[\mathrm{NO}_{2}\right]$ are affected in both approaches. In panel (a) the predicted and measured $\mathrm{N}_{2} \mathrm{O}$ are compared, and panels (b) and (c) demonstrates how the $\mathrm{N}_{2} \mathrm{O}$ profiles need to be vertically shifted in order to obtain an agreement between the model predicted and in situ measured $\mathrm{N}_{2} \mathrm{O}$. Note that the subsidence of higher stratospheric air in the polar vortex (at 09:00 - 13:00 UTC, see Rolf et al., 2015) is not reproduced by the EMAC model. The resulting differences in measured and predicted $\mathrm{N}_{2} \mathrm{O}$ (panel a) indicate a vertical misalignment of up to $4 \mathrm{~km}$ (panel b). The trace gas profiles of $\mathrm{O}_{3}$ and $\mathrm{NO}_{2}$ are shifted together with $\mathrm{N}_{2} \mathrm{O}$. Panel (d) compares $\alpha_{\mathrm{R}}$ based on the different assumptions regarding different profile shapes of the involved gases, and panel (e) the compares the inferred $\left[\mathrm{NO}_{2}\right]$. When forcing the predicted concentrations to the in-situ measured $\mathrm{O}_{3}$ at flight altitude (while keeping the predicted $\mathrm{O}_{3}$ profile fixed elsewhere) the change in inferred $\left[\mathrm{NO}_{2}\right]$ (green dots in panels $\mathrm{d}$ and e) mostly falls into the uncertainty range given by the random error (the gray shaded area in panel e). Evidently the largest discrepancy as compared to the standard run in the simulated $\alpha_{\mathrm{R}}$ and inferred $\left[\mathrm{NO}_{2}\right]$ occurs when the trace gas profiles are altitude shifted (the purple dots in panel $\mathrm{d}$ and $\mathrm{e}$ ). This uncertainty becomes in particular large for flight sections where $\mathrm{O}_{3}$ has strong vertical gradients (e.g. 8:30 - 9:30 UTC and 12:00 - 13:00 UTC). Hence, vertical transport processes which are not covered by EMAC may change the profile shapes in such a way that systematic errors in the scaling method retrieval arise. However, since trace gases such as $\mathrm{NO}_{2}$ and $\mathrm{O}_{3}$ are chemically reactive, the altitude shift carried out here may not reflect the change in trace gas profile shape experienced by $\mathrm{NO}_{2}$ and $\mathrm{O}_{3}$. This is indicated by the fact that the modifications mentioned previously (green dots) do not give rise to large uncertainties in these flight sections, i.e. measured $\left[\mathrm{O}_{3}\right]$ and $\left[\mathrm{NO}_{2}\right]$ do not differ strongly from predictions at flight altitude. 

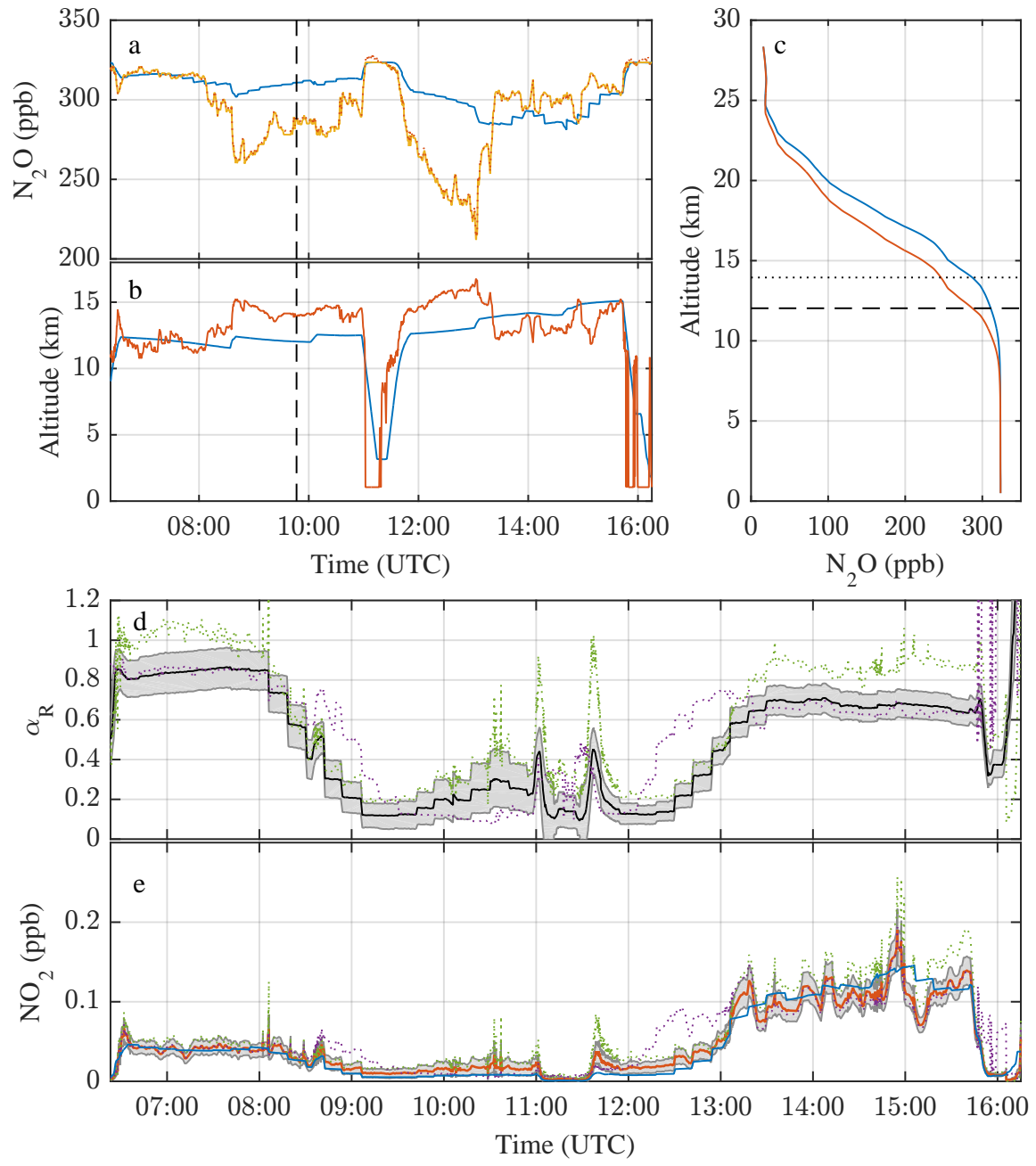

Figure 7: Comparison of sources of potential systematic errors investigated for the HALO flight on 13 September 2012. Panel a: EMAC predicted $\mathrm{N}_{2} \mathrm{O}$ mixing ratio (blue), $\mathrm{N}_{2} \mathrm{O}$ when shifting the predicted EMAC profiles as indicated in panel c (yellow) until predicted $\mathrm{N}_{2} \mathrm{O}$ agrees with in-situ measured $\mathrm{N}_{2} \mathrm{O}$ at flight level (red dots). Panel b: Comparison of aircraft GPS altitude (blue) and apparent altitude (red) where in-situ measured and EMAC predicted $\mathrm{N}_{2} \mathrm{O}$ would agree. Panel c: EMAC predicted $\mathrm{N}_{2} \mathrm{O}$ and altitude shifted $\mathrm{N}_{2} \mathrm{O}$ for the time indicated by the black dashed line in the panels a and $\mathrm{b}$. The broken line marks the aircrafts altitude, the dotted line marks the altitude of the respective $\mathrm{O}_{3}$ mixing ratio in the unchanged EMAC profile. Panel d: $\alpha_{\mathrm{R}}$ (black line) and $\Delta \alpha_{\mathrm{R}}$ due to random errors (gray shaded area). Additionally, $\alpha_{\mathrm{R}}$ resulting from either forcing the simulated to the in-situ measured $\mathrm{O}_{3}$ concentration at flight altitude (green dots) and those resulting from the altitude shifting the $\mathrm{O}_{3}$ profiles (purple dots) are shown. Panel e: Inferred $\mathrm{NO}_{2}$ mixing ratios for (a) the unchanged $\mathrm{O}_{3}$ profile (red line and uncertainty range displayed as gray shaded area), (b) when forcing the EMAC predicted to the measured $\mathrm{O}_{3}$ at flight altitude (green dots), (c) when altitude shifting the profile until the simulated and measured $\mathrm{N}_{2} \mathrm{O}$ agree at flight altitude (purple dots), and (d) for comparison the predicted EMAC $\left[\mathrm{NO}_{2}\right]$ (blue line). 